\title{
Microstructure of Horseshoe Nails Using Neutron Diffraction*
}

\author{
D. J. Goossens \\ Research School of Chemistry and Research School of Physics and Engineering, \\ The Australian National University, ACT 0200, Australia
}

\section{A. J. Studer}

Bragg Institute, Australian Nuclear Science and Technology Organisation, Lucas Heights 2234, Australia

Z. H. Stachurski

Department of Engineering, College of Engineering and Information Technology, The Australian National University, ACT 0200 Australia

\begin{abstract}
Neutron diffraction allows non-destructive testing of the bulk microstructure of mechanical components. The microstructures of horseshoe nails made through three different processes have been explored as a function of position along the nail. Despite all nails being made of similar plain low carbon steel and being process annealed after manufacture, the microstructures are far from the same. Nails made from strip, using a cold forging stamping process, show narrower diffraction peaks indicating a narrower distribution of lattice parameters and also show diffraction peak intensity ratios closer to those expected for unstrained steel. Thus the distribution of the orientation of grains in these nails is closer to that of undistorted steel compared to nails made through the other two processes considered one a drawing from wire, the other a combination of rolling and cold forging. The blades of the drawn nails showed little preferred orientation but the converse was true in the heads. Differing patterns of preferred orientation suggest that the various manufacturing approaches result in substantially different mechanical advantages for the three types of nails, a result in accord with mechanical testing.
\end{abstract}

Keywords: steel microstructure, neutron diffraction, horseshoe nails

* Published in: Journal of Materials Engineering and Performance, 19 (2010) 380-384. DOI:10.1007/s11665-009-9501-z. 


\section{Introduction}

The distribution of residual stresses in metal components can be explored by measuring the resulting strains through their influence on the lattice parameters of the metal as revealed by the shifts in positions of diffraction peaks [1,2]. Similarly, the relative sizes of diffraction peaks, Bragg peaks, from the sample acts as a measure of the population of grains oriented in different directions, which can be compared with that from other samples or with other points in the same sample, and also with that expected for unstrained, ideal material [1]. These microscopic quantities can then be related to the failure modes of the components, helping develop new processes or treatments to improve component performance.

Here, the diffraction patterns of horseshoe nails manufactured through three different processes are compared, both between types of nails and as a function of position along the length of the nail. All nails are made of low carbon plain steel, typically HA3 steel for the process A nails. In all cases the manufacturing processes are performed at room temperature.

The three processes, denoted A, B and C, are outlined as follows:

$\mathrm{A}$ is a stamping process in which the nails are cold forged from strip steel then trimmed and pointed. This process uses mechanical and/or hydraulic presses.

Nails made by process B are made from square wire - headed, drawn, trimmed and pointed.

In $\mathrm{C}$, steel strip is cold rolled lengthways to create a dished profile then the nails are stamped out, trimmed and pointed.

These three processes are very different, and result in very different orientations and populations of grains in the material. All nails were obtained 'as purchased' in retail packaging.

\section{Experimental Procedure}

\section{Neutron Diffraction}

The neutron diffraction experiments were conducted using the High Resolution Powder Diffractometer (HRPD) at the Bragg Institute at the Australian Nuclear Science and Technology Organisation. Low carbon steel is essentially body centred iron, Fe, with a crystallographic unit cell which is cubic, of side length $\sim 2.86 \AA$ (space group is $\operatorname{lm} 3 \mathrm{~m}$ ) [3]. Using neutrons of $1.88345(1) \AA$ wavelength and an angular range of up to $150^{\circ}$ in scattering angle, 2 $\theta$, four Bragg reflections can be observed, the (011), the (002), the (112) and the (022). A smaller wavelength would allow a greater number of reflections to be accessed, but at lower resolution.

Figure 1 shows the parts of a typical horseshoe nail. The nails are of approximately rectangular cross section, varying as shown along the length of the nail. The hardness of the nail is approximately in the range of HRB $88-92$ (Rockwell Hardness on the 'B' scale). In practice the nails are designed and fitted such that the points curl out through the side of the hoof and are twisted off. Because the points are twisted off, their properties are of limited relevance in the longer-term reliability of the nail. This means that mounting the nails by clamping the points results in no loss of useful information.

Figure 2 shows two schematic diagrams of the experiment - viewed from above (a) and the side (b). A cluster of eight nails was arranged in a neutron beam of width greater than the diagonal across the widest part of the sample - approximately $16 \mathrm{~mm}$ - and thickness $1 \mathrm{~mm}$ at the slit. This ribbon beam defined a single slice through the nail, of thickness approximately $1 \mathrm{~mm}$, which allowed the diffraction pattern, and therefore the strains and the grain populations, to be measured as a function of position along the nail by translating the nail cluster vertically between diffraction scans. The sample was rotated in the beam to ensure that all in-plane directions were sampled equally. The use of eight nails not 
only increased the scattering volume (and therefore signal) but meant that the measurement averaged over eight examples of each type of nail, making it more representative of their average structure.

Approximately two hours per diffraction pattern were required for good statistics. Figure 3 shows an example of a scan, in this case from nails of type A. The diffraction peaks are labeled. The flat region close to $120^{\circ}$ shows where a noisy detector has been cut from the pattern (HRPD has 24 detectors). Approximately twenty such diffraction patterns were collected for each nail. One $1 \mathrm{~mm}$ slice was taken every $1.25 \mathrm{~mm}$ through the head of the nail and into the first few millimeters of the blade, and then patterns were taken at $2.5 \mathrm{~mm}$ intervals. In addition, a number of patterns using a $3 \mathrm{~mm}$ slit and $2.5 \mathrm{~mm}$ intervals were taken along the blades of the nails, where the microstructure was expected to be changing more slowly and where the reduced scattering volume due to the narrowing of the nail caused reduced statistics.

\section{Mechanical Testing}

Simple shear tests were performed using an Instron Model 4505. As illustrated in figure 4, a nail was locked into a jig, shearing motion was applied, and the load at yield was measured. Twenty nails of each type were sampled to allow for variability in manufacturing. The samples were sheared at the base of the head, approximately where the hoof and shoe meet, as this is considered the most mechanically critical point.

\section{Results and Discussion}

\section{Neutron Diffraction}

Using a standard data analysis package, each peak in each pattern was independently fitted with a Gaussian. This gave peak height, width, position and therefore intensity. The ratios of the peaks give a measure of preferred orientation, while the positions give the lattice parameter, $a$.

Positions and Widths of Diffraction Peaks: Statistical tests showed that the lattice parameters varied very little between samples and very little within, with all measurements lying within $0.001 \AA$ of the global mean for a given nail; this indicated that within a given $1 \mathrm{~mm}$ slice the distribution of sample strains was approximately centred about zero. Given the geometry of the experiment, the information gained pertains to strains in the plane perpendicular to the length of the nail.

The width of a diffraction peak (see figure 3 for examples of peaks) depends on the distribution of lattice parameters in the sample, the position in $2 \theta$ of the diffraction peak (due to the resolution function of the instrument) and the size of the grains in the sample; for steels such as these, the grain sizes are large (approx. $10 \mu \mathrm{m}$ ) and are not expected to have substanital influence on Bragg peak widths, so broading must be interpreted in terms of strain. The same instrument on the same settings was used for all samples, so this allows the relative widths (full width at half maximum, or FWHM) of the peaks to be compared between samples. This comparison is summed up in table 1, which shows the average width (in degrees) of each peak for each sample. The widths varied little over the length of the nails, although there tended to be a small decrease from head to point due to (a) the smaller volume of sample near the points resulting in more homogeneous strains and (b) the more complex shaping required to produce the head and neck causing more lattice parameter shifts associated wit plastic deformation and higher dislocation densities. Smaller full widths indicate that the distribution of interatomic spacings in sample A is narrower, indicating 
lower strains. Hence while none of the processes shifts the centre of the distribution of interatomic distances within a slice, the range of such spacings is narrower in nails produced through process A, indicating they have lower strains. Despite the very different processes, the peak widths in samples B and C were almost identical.

Intensities of Diffraction Peaks: The relative intensities of the diffraction peaks act as measurements of the preferred grain orientation in the sample. These ratios can be compared between samples, as a function of position within a sample, and with the expected ratios for an unstrained example of BCC iron as calculated from the ideal structure. Given that the samples were rotated in the beam, preferred orientation within the plane of the ribbon beam will be averaged out. Hence this experiment ia a measurement of preferred orientation relative to the long axis of the nail - in other words, axial preferred orientation. We refrain from using the term 'texture' as this implies true three-dimensional texture mapping as would be presented in a pole figure.

Such results are shown in figures 5a, b and c for samples A, B and $\mathrm{C}$ respectively. To make these comparisons while allowing for the different scattering volumes of the samples and at the different points along the samples, all experimental results were divided through by the intensity of the (011) peak at that position along the nail. Error bars increase as the scattering volume decreases, which occurs as the distance from the head increases.. Indicative error bars are shown on the figure. For comparison, peak intensities (intensity of peak $h k l$ is denoted $I_{h k l}$ ) for a sample without preferred orientation were calculated based on the structure factors for the reflections. These are plotted on each of the plots in figure 5, with horizontal lines indicating the calculated ratios: solid lines indicating the ratio $I_{112} / I_{011}$ (the (112) has almost exactly the same expected intensity as the (011)), dashed lines indicating $I_{022} / I_{011}$ and dotted lines indicating $I_{002} / I_{011}$.

It is immediately apparent that the preferred orientation depends very heavily on position along the nail's length. This is most pronounced in samples $\mathrm{B}$ and $\mathrm{C}$, in which peaks crossover such that the $(022)$ relative to the $(011)$ goes from being stronger than the $(002)$ in the head and neck of the nail to being weaker in the blade. In the blade of the type $C$ nail the (002) is virtually as strong as the (011), even though it would be expected to be no more than a third the intensity of the (011) in a sample without preferred orientation. In sample B all reflections are much closer to their expected ratios in the blade of the nail, though the (112) is rather strong compared to the (011).

Sample A shows no crossover at all; indeed, all four peaks are always ranked correctly compared with their expected intensity, with the biggest anomaly being that the (112) is never as strong as the (011), when it should be virtually the same were the nail without preferred orientation. Again, rapid changes can be seen near the top of the blade, with a sharp dip in the (112) intensity and less sharp but pronounced features in the (022) and (002).

These patterns of preferred orientation reflect the fact that the head and neck are the most heavily shaped and processed parts of the nail. A rapid change in preferred orientation implies a rapid change in grain populations near the base of the neck and for the top few millimeters of the blade. This implies a region of high density of grain boundaries, which would generally be considered to contribute to toughening of the component in that region. (Smaller grain size would mean higher yield strength - the Hall-Petch effect [4].)

In summary, nails of type A are stamped out from normalized strip. There is little deformation in the shank. Grains appear the same size as for other nails (approx. $10 \mu \mathrm{m}$ ) with no elongation. Therefore we see the near normal diffraction pattern for distances $>5 \mathrm{~mm}$ from the top of the head (the shank). 
Nails of type B are made from wire that is drawn and shaped. Grain sizes are approximately the same as in A, but slightly elongated in the drawing direction, giving the enhanced (112) reflection for distances $>5 \mathrm{~mm}$ (in the shank). The resulting motion of dislocations on slip planes has also increased the intensity of (022) relative to (002). The pattern in the head section is similar to that in A, except the intensity of (112) has increased due to the plastic deformation.

Nails of type $C$ are cold rolled lengthwise. $<100>$ and $<111>$ directions rotate towards the compression axis. This will bring the normal to the (112) planes into greater concentration, hence the increased intensity.

Table 1 shows that samples B and $\mathrm{C}$ showed the broadest peaks, and the broadening is very similar in magnitude. However, figure 5 shows that the type $C$ nails show a less pronounced change in preferred orientation at the neck/blade juncture than the other nails, suggesting a more continuously varying population of grain orientations, but that the grains themselves are equally strained in B and C, and less strained in A. The change in preferred orientation in type A nails is more localised than in the others, and does not extend down into the neck and blade. As it is in the neck and upper blade that the nail is expected to fail [5], this is likely to be detrimental to the mechanical properties in real world use.

Hence, varying patterns of evolution of preferred grain orientation have been successfully identified in the three types of nails. These patterns relate to one of the most common failure modes of horseshoe nails - the heads' shearing off [5]. The neck/blade join is the region of the nail under the greatest mechanical demand due to the large shearing forces experienced during galloping as the hoof spreads laterally on impact with the ground. Withstanding this requires high toughness, which these results suggest should be greater in the type $\mathrm{B}$ and $\mathrm{C}$ nails (the preferred orientation changes more in the relevant part of the nail, indicating more grain boundaries). Having said that, the degree of plasticity will also be crucial. The region of changing preferred orientation extends down into the blade of the nail in types B and C, particularly $\mathrm{C}$, and will contribute to increasing the toughness of the top section of the blade which will also contribute to nail longevity.

\section{Mechanical Testing}

The load at yield of each nail type and its error (two standard deviations), normalised for cross-sectional area, are shown in table 1. Nails of type A show the lowest shear strength, as might be expected based on the neutron diffraction results. Types $\mathrm{B}$ and $\mathrm{C}$ show very similar values per unit cross sectional area, with $\mathrm{C}$ slightly more resistant to the shearing forces. This must be interpreted in the light of the standard deviations, which show that type $\mathrm{C}$ is clearly much more resistant to shear than type A, but that type B shows by far the greatest variability, and so while it is most likely better than A and slightly lower in performance than $\mathrm{C}$, the spread in values militates against a definite conclusion. Nevertheless, it can be concluded that the mechanical testing is in accord with the results from neutron diffraction, although it does not offer the insight into the mechanism for the trends in the results.

It was also found that type A nails were most likely to have the head completely severed at the yield stress, whereas type $\mathrm{C}$ and particularly type B nails showed greater plastic deformation, suggesting they are less prone to sudden failure. This accords with results inferred from neutron diffraction which showed that type $\mathrm{C}$ is expected to be more plastic than A, while type B shows a larger region of the nail is expected to be toughened by high grain boundary density, again making it more mechanically strong than A.

\section{Conclusions}

Position dependent neutron diffraction can be used to non-destructively explore the preferred 
orientation in manufactured components to allow correlation of their microstructure with their mechanical properties. This also eliminates the alterations to the microstructure that can arise when trying to gain similar results through sectioning of the part. We have examined horseshoe nails made through three different processes and successfully found large differences in their microstructure, and a large dependence of the preferred orientation of the grains on position along the nail. One process (C) gives preferred orientation which changes substantially more slowly through the most mechanically crucial section of the nail, and extends over a large region within the nail. Further, another process (A) shows change in preferred orientation to be highly localised, and this has an undesirable effect on the mechanical properties.

\section{References:}

[1] M.T.Hutchings, P.J.Withers, T.M.Holden and T.Lorentzen, Introduction to the Characterization of Residual Stress by Neutron Diffraction, CRC Press, 2005.

[2] X.-L. Wang, The Application of Neutron Diffraction to Engineering Problems, JOM-J. Min. Met. Mat. Soc., Vol 58 (No 3), 2006, p 52-57.

[3] P. Villars and L.D. Calvert, Pearson's Handbook Of Crystallographic Data For Intermetallic Phases, 2nd ed., vol. 3, ASM International, USA, 1991.

[4] H. M. Rosenberg, The Solid State, 3rd ed., OUP, Oxford, UK, 1989, p. 74.

[5] http://www.horseshoes.com/forums 


\section{Figure captions:}

Figure 1: Schematic diagram of a horseshoe nail from two viewpoints, noting the major segments.

Figure 2a: Top view of the experimental setup for neutron diffraction. A cluster of eight nails is rotated in a ribbon beam while the data is collected. Figure 2b: Experimental setup, side view. The ribbon beam is $1 \mathrm{~mm}$ thick at the sample. A pattern is collected then the nail is translated down by $1.25 \mathrm{~mm}$ and another pattern is collected.

Figure 3: Neutron diffraction pattern of nails of type A, Bragg peaks indexed. Flat region shows where an unreliable detector has been excised.

Figure 4: Schematic diagram of the shear experiment. The nail (black polygon) is clamped against the lower block while the blade is driven down as arrowed and the forces recorded.

Figure 5: Scattering intensity normalized by that of the (011) reflection as a function of position along the length of each type of nail. Triangles $(\boldsymbol{\Delta}, \triangle)$ indicate the (112) reflection, circles $(\boldsymbol{O}, \mathbf{O})$ indicate the $(002)$ reflection and boxes $(\boldsymbol{\square}, \square)$ indicate the $(022)$ reflection. Filled points were measured using the $1 \mathrm{~mm}$ slit, open the $3 \mathrm{~mm}$ slit. Representative error bars are shown. Solid lines are guides for the eye. Dotted vertical lines show where the neck joins the blade. Solid horizontal lines gives the calculated ('calc') expected intensity of the (112) relative to the (011), the dashed lines give that for (022) and horizontal dotted the (002) (examples are noted on (b)). 
Table 1: Average full width at half maximum of each Bragg peak, with uncertainty in the last decimal place in parentheses. For all peaks process A gives the narrowest distribution.

\begin{tabular}{|c|c|c|}
\hline Sample & Peak & $\begin{array}{c}\text { Average } \\
\text { FWHM }\left({ }^{\circ}\right)\end{array}$ \\
\hline & 011 & $0.26(2)$ \\
A & 002 & $0.27(2)$ \\
& 112 & $0.27(2)$ \\
& 002 & $0.45(4)$ \\
\hline & 011 & $0.29(2)$ \\
B & 002 & $0.37(3)$ \\
& 112 & $0.39(2)$ \\
& 002 & $0.59(9)$ \\
\hline & 011 & $0.30(3)$ \\
C & 002 & $0.39(5)$ \\
& 112 & $0.41(4)$ \\
& 002 & $0.60(1)$ \\
\hline
\end{tabular}


Table 2: Measured yield strengths in shear of the three rtypes of horseshoe nails (average values for 20 samples each).

\begin{tabular}{|l|l|}
\hline Horseshoe nail & Stress at yield point $\left[\mathrm{kN} / \mathrm{mm}^{2}\right]$ \\
\hline A & $0.36 \pm 0.03$ \\
\hline B & $0.41 \pm 0.05$ \\
\hline C & $0.42 \pm 0.03$ \\
\hline
\end{tabular}




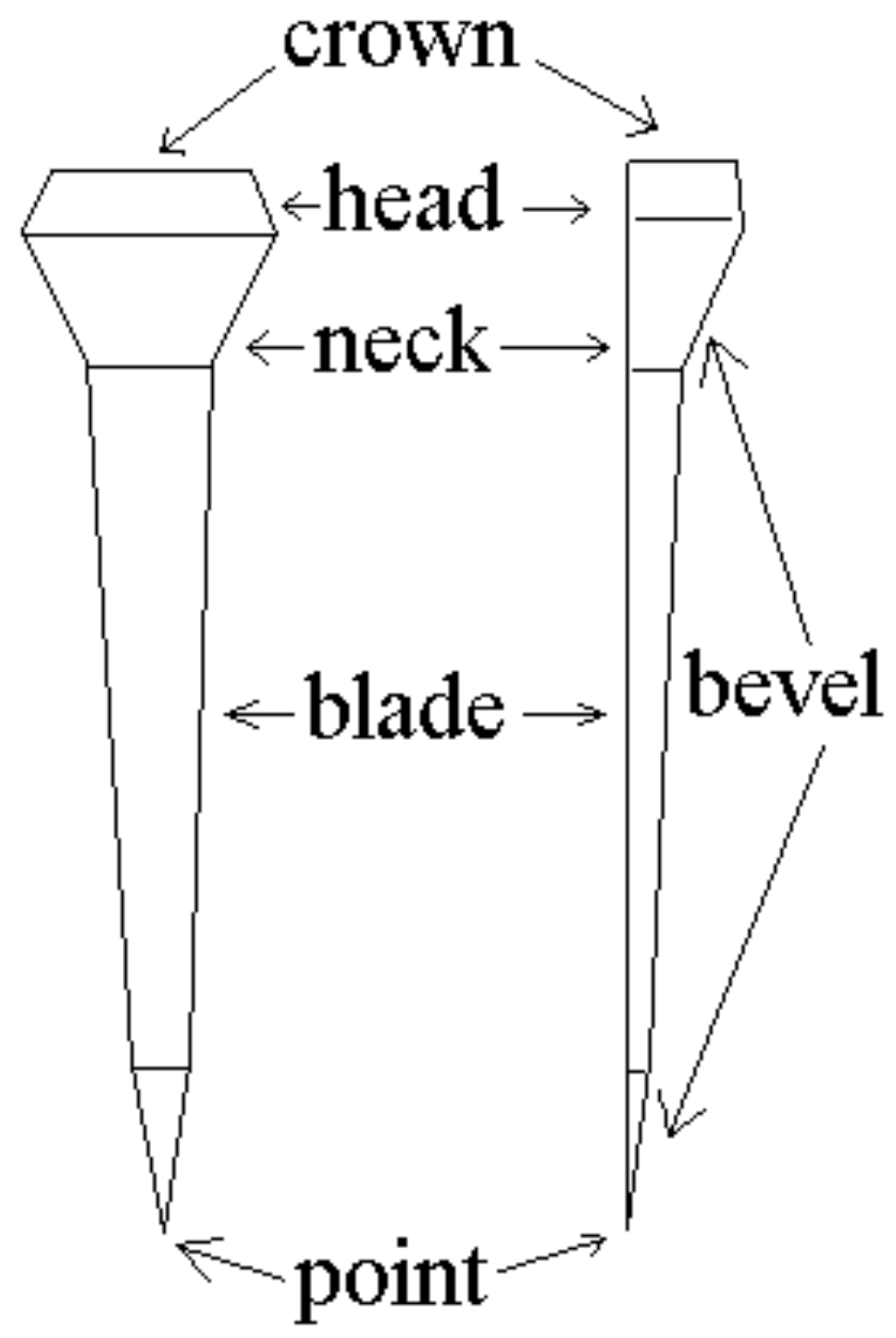

Figure 1

Goossens_Fig1.tif 


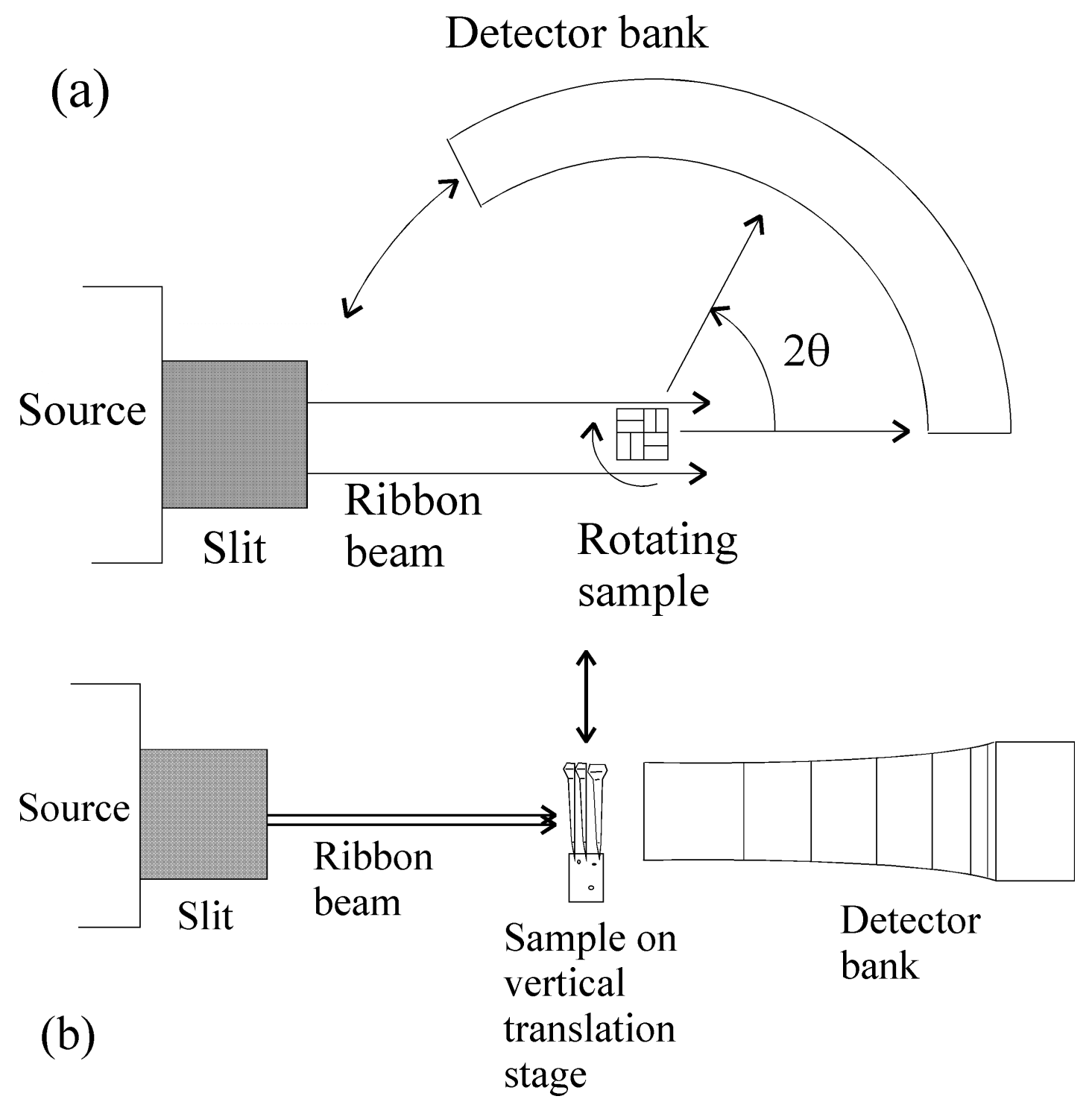

Figure 2

Goossens_Fig2b.tif

Goossens_Fig2a.tif 


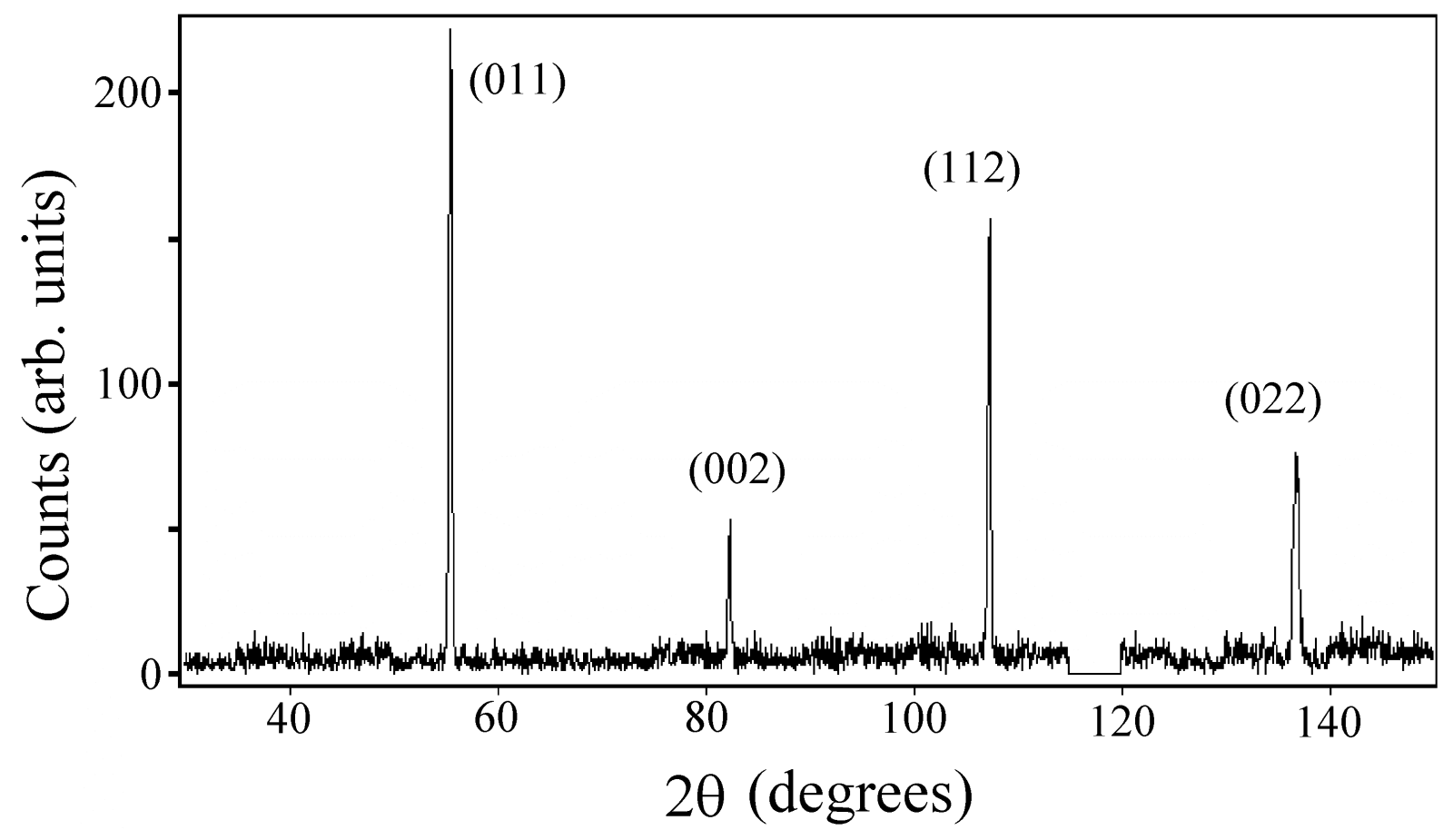

Figure 3

Goossens_Fig3.tif 


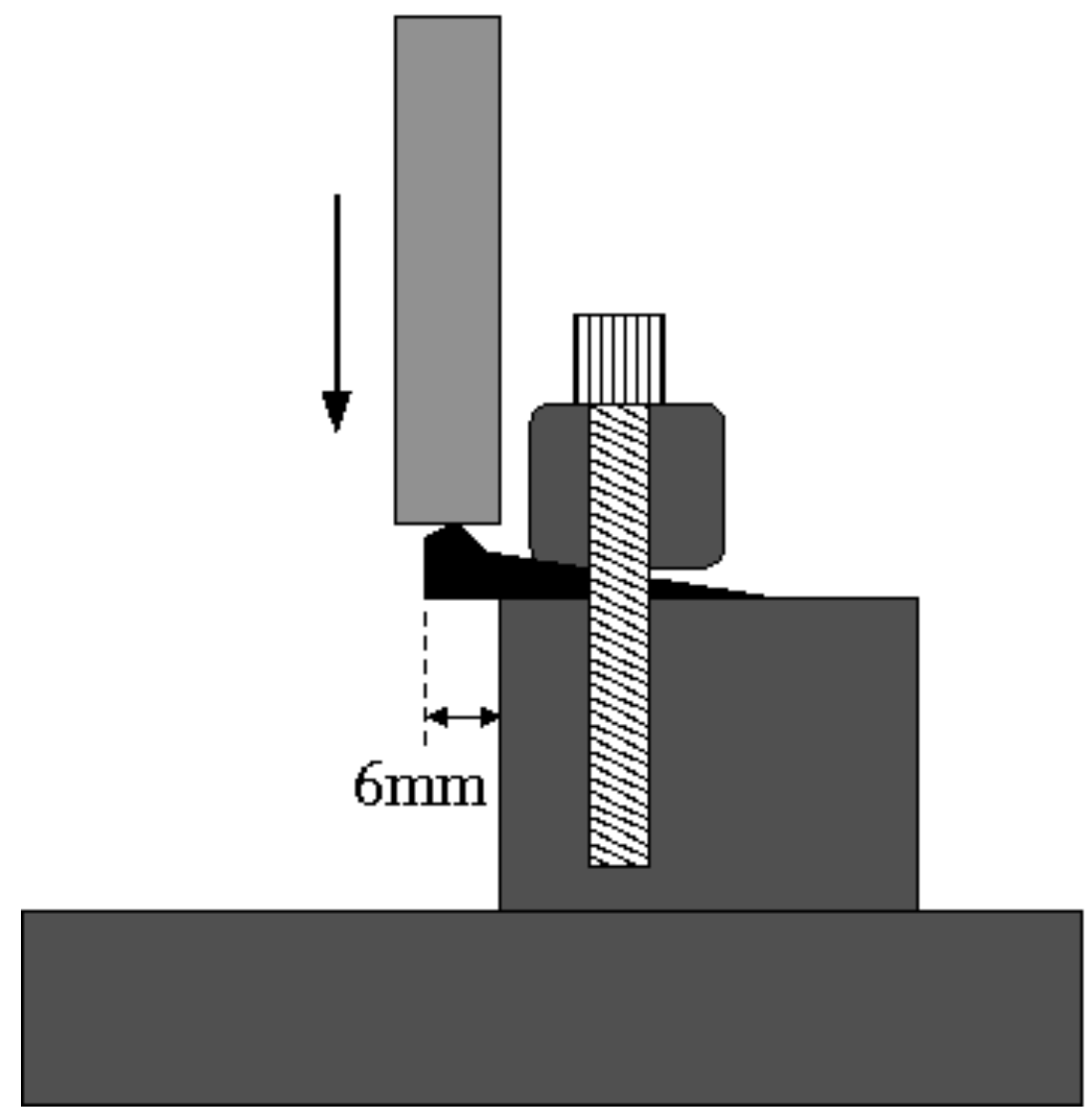

Figure 4.

Goossens_Fig4_u2.tif 


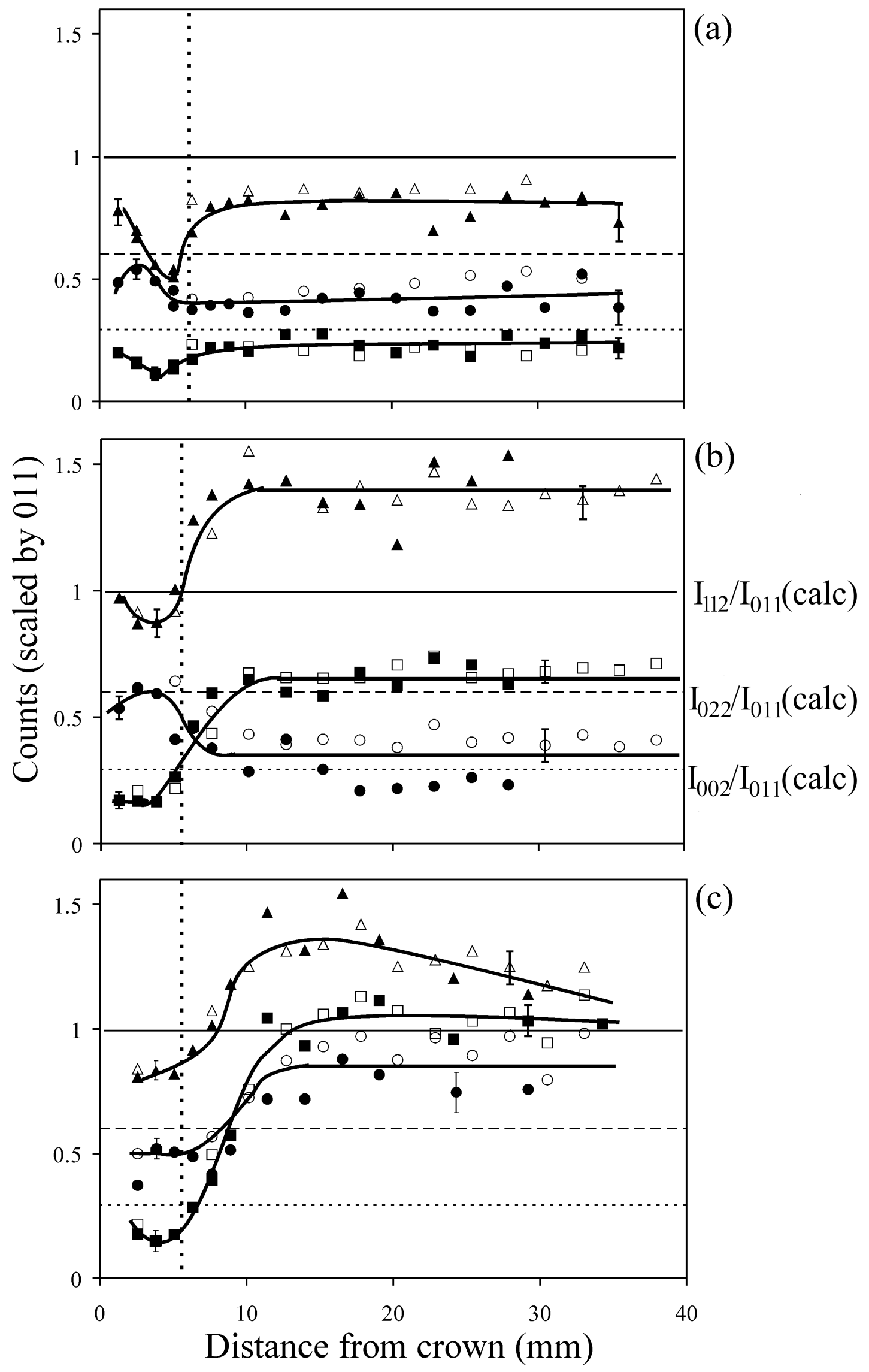

Figure 5: Goossens_Fig5_2009.tif 